\title{
Josephson tunnel junction microwave attenuator
}

Koshelets, V. P.; Shitov, S. V.; Shchukin, A. V.; Baryshev, A. M.; Filippenko, L. V.; Fischer, Gerd Michael; Mygind, Jesper

Published in:

Applied Physics Letters

Link to article, DOI:

$10.1063 / 1.110203$

Publication date:

1993

Document Version

Publisher's PDF, also known as Version of record

Link back to DTU Orbit

Citation (APA):

Koshelets, V.P., Shitov, S. V., Shchukin, A. V., Baryshev, A. M., Filippenko, L. V., Fischer, G. M., \& Mygind, J. (1993). Josephson tunnel junction microwave attenuator. Applied Physics Letters, 63(23), 3218-3220.

https://doi.org/10.1063/1.110203

\section{General rights}

Copyright and moral rights for the publications made accessible in the public portal are retained by the authors and/or other copyright owners and it is a condition of accessing publications that users recognise and abide by the legal requirements associated with these rights.

- Users may download and print one copy of any publication from the public portal for the purpose of private study or research.

- You may not further distribute the material or use it for any profit-making activity or commercial gain

- You may freely distribute the URL identifying the publication in the public portal

If you believe that this document breaches copyright please contact us providing details, and we will remove access to the work immediately and investigate your claim 


\title{
Josephson tunnel junction microwave attenuator
}

\author{
V. P. Koshelets, S. V. Shitov, A. V. Shchukin, A. M. Baryshev, \\ and L. V. Filippenko \\ Institute of Radio Engineering and Electronics, RAS, Moscow 103907, Russia
}

G. M. Fischer and J. Mygind

Technical University of Denmark, B 309, DK-2800 Lyngby, Denmark

(Received 23 July 1993; accepted for publication 1 October 1993)

\begin{abstract}
A new element for superconducting electronic circuitry-a variable attenuator - has been proposed, designed, and successfully tested. The principle of operation is based on the change in the microwave impedance of a superconductor-insulator-superconductor (SIS) Josephson tunnel junction when dc biased at different points in the current-voltage characteristic. Both numerical calculations based on the Tien-Gordon theory and $70-\mathrm{GHz}$ microwave experiments have confirmed the wide dynamic range (more than $15-\mathrm{dB}$ attenuation for one stage) and the low insertion loss in the "open" state. The performance of a fully integrated submillimeter receiver circuit which comprises a flux-flow oscillator (FFO) as local oscillator, a superconducting variable attenuator, and a microwave SIS detector with tuned-out capacitance is also reported.
\end{abstract}

Integrated superconducting local oscillators (LO) for millimeter and submillimeter superconductor-insulatorsuperconductor (SIS) mixer receivers have been the subject of intensive investigations during recent years and different types of superconducting LO's have been successfully tested..$^{1-3}$ In order to optimize the performance of the SIS mixer the applied LO power must be adjusted with an accuracy of about $1 \mathrm{~dB}$. This is an inherent problem for synchronous arrays of small Josephson junctions ${ }^{1}$ and resonant soliton oscillators [long low-damping Josephson tunnel junctions (Ref. 3)], whereas for flux-flow oscillators (FFO) the microwave power may be adequately tuned ${ }^{2,3}$ by changing the dc current bias and/or the applied magnetic field. Unfortunately, such adjustments usually result in changes of both frequency and linewidth of the emitted power. To provide on-chip fine tuning of the amplitude of high frequency signals we have introduced a new integrated circuit element-the superconducting attenuator.

The principle of operation of the attenuator is based on the change in the microwave impedance, $Z(V)$, of a small Josephson tunnel junction when biased at different dc voltages, $V$. This dependence is shown in Fig. 1 for $f \approx e V_{g}$ $10 \mathrm{~h}$ together with the model current-voltage characteristic $(I V C)$ which was used in the calculation of $\operatorname{Re} Z$ based on Tucker's theory ${ }^{4}$ for small tunnel junctions. For operational frequencies less than the gap frequency, $f<e V_{g} / h$, where $V_{g}$ is the gap voltage, one can distinguish between three regions on the $I V C$. For $V \gg V_{g}$ the real part of the junction impedance, $\operatorname{Re} Z$, is equal to the normal state resistance, $R_{n}$. Re $Z \gg R_{n}$ for $|V|<(V g-h f / e)$ and finally $\operatorname{Re} Z<R_{n}$ for $V \approx V_{g}$. This simplified scheme assumes that the critical current is suppressed and the capacitance of the junction is resonated out.

The attenuator junctions which are placed in the (microstrip) transmission line between the signal source and the detector, may be connected in series or in parallel; in both cases $R_{n}$ should approximately match the characteristic impedance of the line. The insertion loss is high (at- tenuator in the "closed" state) for $|V|<\left(V_{g}-h f / e\right)$ if series connected, and for $V \approx V_{g}$ if connected in parallel; the "open" state has the opposite combination of bias conditions. The microwave power level must be so low that it itself does not influence $\operatorname{Re} Z(V)$.

A particular series of integrated microstrip circuits were designed and tested to verify the performance of the attenuator. The equivalent diagram and the layout of one of the circuits are shown in Fig. 2. The 70-GHz microwave signal enters through a fin-line antenna and a microstrip quarter-wave transformer (symbolized by $T_{1}$ in Fig. 2 ). The circuit shown has three identical attenuator sections: ${ }^{5}$ $P$ is a parallel attenuator ( $P$-att), while the two sections, $\mathbf{S}_{1}$ and $S_{2}$, together form a series attenuator (S-att). The $\mathrm{W}=40 \mu \mathrm{m}$ wide a microstrip line has approximately a $1-\Omega$ characteristic impedance. Each section consists of two SIS junctions (area $25 \mu \mathrm{m}^{2}, R_{n}=4-8 \Omega$ ), which are connected in series for rf signals and in parallel at dc. The junction capacitances are resonated out at the signal frequency by a small inductance. The frequency range in which the capacitances are compensated is determined by the value of $\gamma=2 \pi f C \operatorname{Re} Z$ and depends on the attenuator

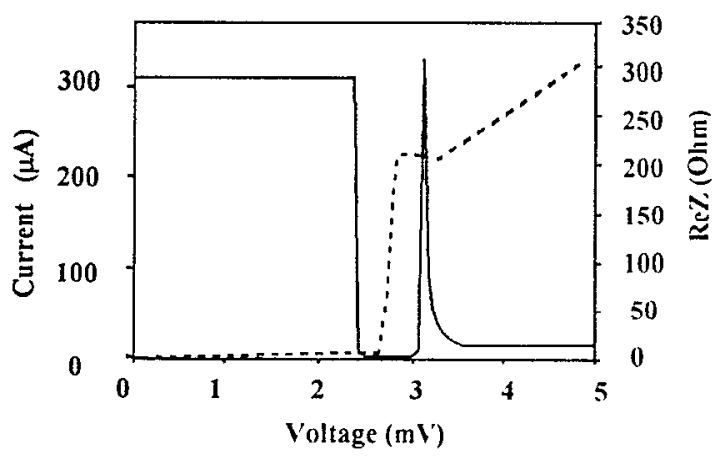

FIG. 1. Real part of the microwave impedance, $\operatorname{Re} Z$, of the SIS junction as function of the bias voltage, $V$, calculated at frequency $f=e V_{g} / 10 h$ $=70 \mathrm{GHz}$. The model $I V C$, with normal state resistance $R_{n}=15 \Omega$, used in the simulation is shown by the dashed line. 


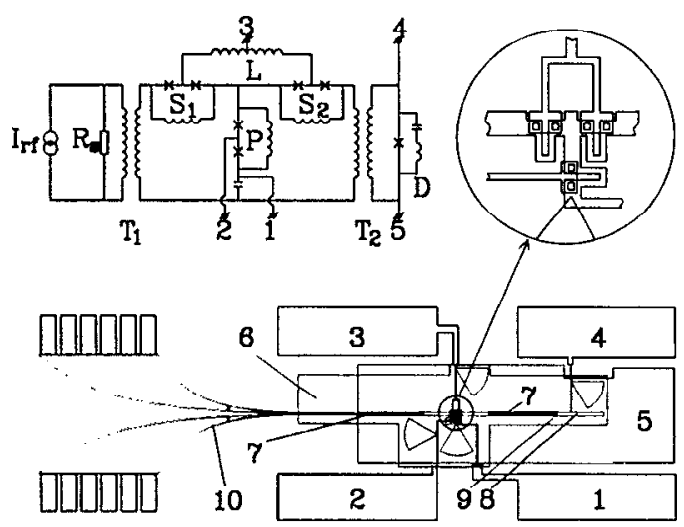

FIG. 2. Equivalent diagram and layout of the integrated attenuator circuit. 1-5-contact pads; $6-\mathrm{SiO}_{2}$ isolation; 7-microwave quarter-wave transformers, 8-tuning-out microstrip stub; 9-SIS detector; 10-fin-line antenna.

bias $\left(\gamma \approx 3\right.$ for $\left.\operatorname{Re} Z=R_{n}\right)$. The two S-att sections are dc biased in parallel via the large inductance $L$. Another microwave transformer, $T_{2}$, leads to the small detector SIS junction (area $5 \mu \mathrm{m}^{2}, R_{n}=20 \Omega$ ). Its capacitance is tuned out by the inductance of a microstrip stub.

The microcircuits had three superconducting $\mathrm{Nb}$ layers and two double $\mathrm{SiO}_{2}$ layers, and were fabricated with a technology developed for SIS and rapid signal flux quantum (RSFQ) devices. ${ }^{6}$ All junctions were high-quality trilayer $\mathrm{Nb}-\mathrm{AlO}_{x}-\mathrm{Nb}$ tunnel junctions with critical current density $j_{c}=2 \times 10^{3} \mathrm{~A} \mathrm{~cm}^{-2}$ and subgap to normal state resistance ratio, $R_{j} / R_{n}>20$.

Figure 3(a) demonstrates the action of the series attenuator (S-att) at $f=74.6 \mathrm{GHz}$ for moderate input power levels; the $I V C$ of the S-att, measured between terminals No. 1 and No. 3 on Fig. 2, is shown in the insert. The $I V C$ 's of the detector junction were recorded with the $\mathrm{S}$-att biased at the four different points marked in the insert, and clearly there is a significant change in the detected microwave power.

At point $\mathrm{A}\left(V \approx V_{g}\right)$ the S-att is open $\left(\alpha \equiv e V_{r f} / h f\right.$ $\approx 2.2)$, while at point $\mathrm{D}(V \simeq 0, \alpha \sim 0.03)$ only marginal microwave power reaches the detector as seen from the $I V C$, being very similar to the unpumped curve. The observed voltage amplitude can be reduced by more than 70 times corresponding to at least $17-\mathrm{dB}$ decrease in power level (corrected for the change in the microwave impedance of the detector) by adjusting the dc bias of the S-att.

With large input power the attenuator "saturates" [see Fig. 3(b)]. The change in $\operatorname{Re} Z$ is smaller and consequently the dynamic range of the attenuator becomes considerably lower (only about $5 \mathrm{~dB}$ ). Therefore the actual power level of the FFO must be taken into account in the design of a practical attenuator.

In order to improve the experimental accuracy we modulated the microwave power and measured the detector response at $V \approx V_{g}-h f / 2 e$ by lock-in technique. The experimental results are shown in Fig. 4(a) by a solid line (1). One can estimate from curve (1) that the attenuation level is about $20 \mathrm{~dB}$, which is close to the result derived from Fig. 3(a). The corresponding IVC of the S-att is the
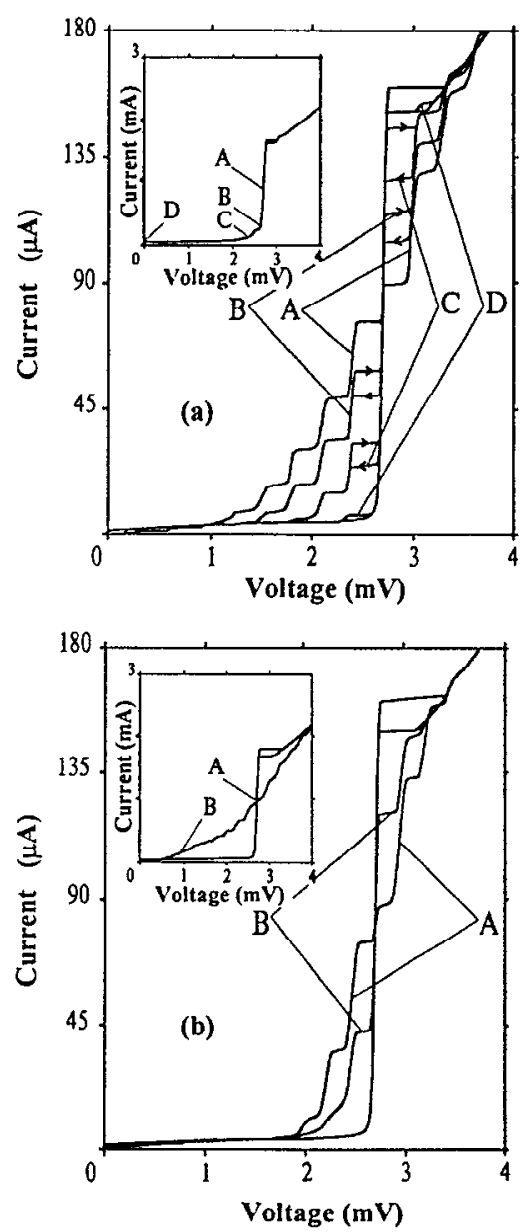

FIG. 3. IVC's of the SIS detector at different bias voltages at input frequency $f=74.6 \mathrm{GHz}$ showing action of the series attenuator (S-att) at low level (top) and high level (bottom) of the input power. Insert shows the corresponding $I V C$ 's of the S-att.

dashed line (3). The result of the numerical calculations of the detector response, using the $\mathrm{S}$-att model $I V C$, is shown by the dashed-dotted line (2), which is normalized to the experimental curve at $V>V_{g}$. The best fit to the experimental curve is obtained when also taking into account the incomplete tuning-out of the junction capacitance $\left(C_{u n t} / C\right.$ $\approx 0.25$ ). Quantitatively there is good agreement between theory and experiment; the discrepancy near $V \simeq V-g$ probably is due to the imperfect approximation of the experimental $I V C$, and/or instabilitics of the opcration point on the "knee" structure. The difference in the response at $V<V_{g}$ might be explained by a microwave signal bypassing the "closed" attenuator.

Figure 4(b) shows the action of the parallel attenuator (P-att). With the experimental parameters of the attenuator junctions and the microstrip lines the dynamic range of the P-att is lower than for the S-att, nevertheless an attenuation of about $7.5 \mathrm{~dB}$ has been obtained. The theoretical response (curve 2), which was calculated with the same fitting parameters as used for the S-att, agrees well with experiment. It should be noted that one cannot simply add the individual insertion losses when operating both the series and parallel attenuators in the circuit.

In order to achieve ultimate performance of a SIS 

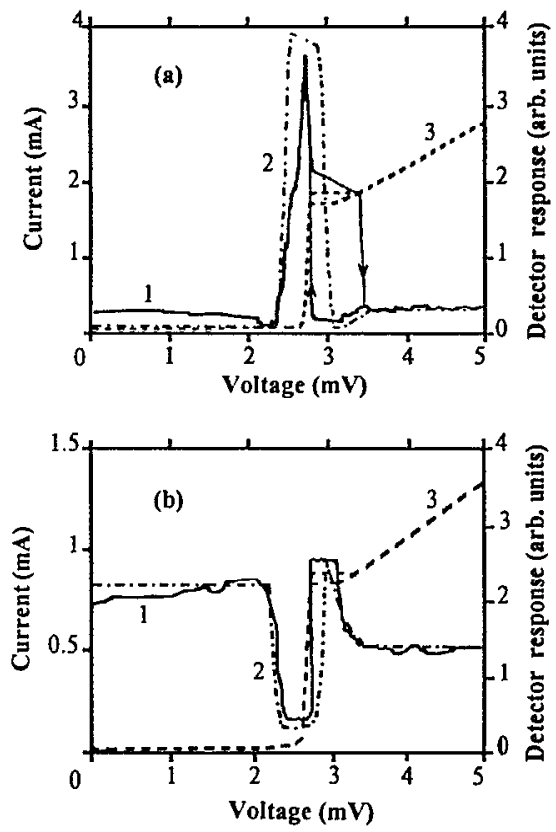

FIG. 4. Experimental (1) and simulated (2) detector response vs bias voltage of the series (a) and parallel (b) attenuators. Model IVC's of the corresponding attenuators are shown by the dashed curve (3).

mixer receiver the power of the LO should be carefully controlled without changing its frequency and linewidth. To meet this requirement at preset frequencies in the mmwave band we have designed and investigated fully integrated receiver circuits which comprise a FFO (as on-chip LO), a SIS microwave detector, several matching elements, and an adjustable superconducting high frequency attenuator. Figure 5 shows the action of the integrated attenuator at frequency $325 \mathrm{GHz}$. Because of nonoptimal design the attenuator junctions were subjected to large signal amplitudes and consequently only a relatively small dynamic range and a considerable insertion loss in "open" state were observed. Numerical simulations, however, predict that the performance of the integrated attenuator circuit may be considerably improved.

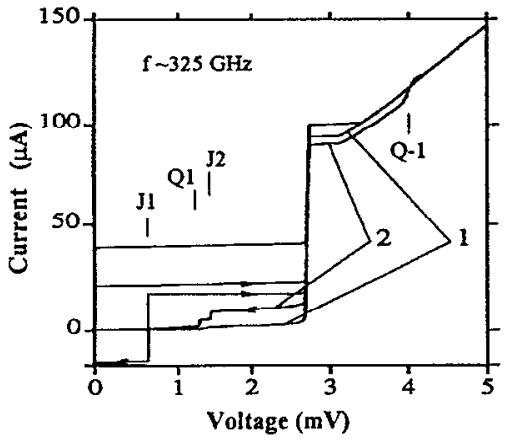

FIG. 5. IVC of a SIS detector at two levels of applied power from an on-chip integrated flux-flow oscillator (FFO, $f=325 \mathrm{GHz}$ ). The power was adjusted by a superconducting attenuator inserted in the line between the FFO and the detector. Symbols $J_{N}$ and $Q_{N}$ refer to the $N^{\prime}$ th rf-induced Josephson step and quasiparticle step, respectively.

The present results confirm the feasibility of the proposed adjustable superconducting microwave attenuator. Its wide dynamic range (more than $15 \mathrm{~dB}$ ) and small insertion loss in the "open" state have been demonstrated in experiments and by numerical simulations. The proposed attenuator has potential applications in integrated $\mathrm{mm}$ wave receivers as on-chip precision attenuator and microwave power modulator, and may be used up to the gap frequency with a recent design of a parallel biased array. ${ }^{7}$

Research supported in part by the Russian Program of Fundamental Research, Contract No. 92-02-3484, and by NATO Linkage Grant No. 921040.

${ }^{1}$ B. Bi, S. Han, J. E. Lukens, and K. Wan, IEEE Trans. Appl. Supercond. 3, 2303 (1993).

${ }^{2}$ V. P. Koshelets, A. V. Shchukin, S. V. Shitov, and L. V. Filippenko, IEEE Trans. Appl. Supercond. 3, 2524 (1993).

${ }^{3}$ Y. M. Zhang, D. Winkler, and T. Claeson, Appl. Phys. Lett. 62, 25 (1993).

${ }^{4}$ J. R. Tucker and M. J. Feldman, Rev. Mod. Phys. 4, 1055 (1985).

${ }^{5}$ An. B. Ermakov, V. P. Koshelets, S. A. Kovtonjuk, and S. V. Shitov, IEEE Trans. Magn. MAG-27, 2642 (1991).

${ }^{6}$ V. P. Koshelets, S. A. Kovtonjuk, I. L. Serpuchenko, and L. V. Filippenko, IEEE Trans. Magn. MAG-27, 3141 (1991).

${ }^{7}$ S. V. Shitov, V. P. Koshelets, S. A. Kovtonjuk, and A. M. Baryshev, IEEE Trans. Appl. Supercond. 3, 2261 (1993). 